\title{
Total Body Water Changes Using Segmental Bioimpedance in Healthy Population with Similar Anthropometry
}

\author{
J. Rosell-Ferrer ${ }^{1}$, L. Nescolarde ${ }^{1}$, and J. Elvira ${ }^{2}$ \\ ${ }^{1}$ Technical University of Catalonia/Electronic Engineering Department, Barcelona, Spain \\ ${ }^{2}$ NTE S.A, Barcelona, Spain
}

\begin{abstract}
Electrical bioimpedance was measured in 7 body segments, and also with the standard right-side method, using 11 electrodes at 6 different frequencies in 8 healthy male subjects with similar anthropometry. Our objective was to determine the capability of segmental bioimpedance measurements to estimate small changes of water on each segment (TWsegi) and total body water (TBW) in comparison with the standard right-side method. Water was also estimated with ${ }^{40} K$ and DXA. Volunteers were measured before and after a $3.5 \%$ water load of their individual TBW. The expected TBW mean increment after water load was $1.45 \mathrm{l}$. The estimator with lower Standard Error SE was the weight of the subject (0.15 l). For impedance methods, the $\mathrm{SE}$ of the segmental method was 0.94 I vs. 1.41 I for right-side. Segmental volume changes obtained by DXA and Impedance compared with expected values showed maximum differences of almost $2 \mathrm{I}$ for DXA and $0.5 \mathrm{I}$ for $\mathrm{Z}$ in the abdomen. In conclusion, in a healthy sample with similar anthropometry, such as astronauts and athletes, the use of a segmental impedance method improves the accuracy of the right-side method to estimate TBW. Changes in water segments estimated by impedance where more close to expected values than using DXA.
\end{abstract}

Keywords - segmental, whole-body, bioimpedance, total water.

\section{INTRODUCTION}

The standard whole-body or right-side (RS) impedance measurement for estimated of body composition performed by using a multi-frequency (MFBIA) bioimpedance measurement estimate TBW using the real part of impedance (R) at high frequencies, and the extra-cellular volume using $\mathrm{R}$ measured or estimated at low frequencies. The basic assumption is that at low frequency the current travels through the extra-cellular water space, while at high frequency it travels between all spaces: intra-cellular and extra-cellular [1].

Hoffer et al [2] used this model to estimate TBW using the measurement of right-side impedance, with the assumptions that the human body physically approximates a cylinder, where length (L) equals the body height (Ht) and given that body water space has uniform resistivity. Several equations described by Ellis [3] included one or more terms related to the measured impedance, fundamentally $(\mathrm{Ht})^{2} / \mathrm{R}$, plus additional anthropometric terms. In general, these estimators are more likely to violate hypotheses, especially those regarding tissue hydration [3].

Since Nyboer 1940 fins the actuality numerous investigations [4-7] have analyzed the feasibility of segmental bioimpedance measurement in the determination of segmental volumes and regional fluid shifts to overcome the limitations of right-side impedance methods.

The main objective of this work is to determine the capability, of segmental bioimpedance measurements, for estimating small changes of total water, in several segments and total body water in whole-body, in healthy humans with similar anthropometry. We propose equations for estimating changes of total water in each segment and in the wholebody. The proposed equations reduce the number of variables used to a minimum, and we exclude weight to allow measurements in microgravity. We compare the results using bioimpedance with the expected changes on body water due to the water load and the changes detected using the DXA scan system. We also compare the results using RS impedance versus an estimation of the whole-body volume using the addition of segmental volumes. The a priori advantages of the bioimpedance technique, especially for aero-space and sport applications, are its simplicity and the low volume and weight of the required measurement instrumentation.

\section{MAterial AND METHODS}

\section{A. Subjects}

Eight male health subjects were selected to match the anthropometry and age of astronaut candidates: (23-29 yr, 74$84 \mathrm{~kg}, 179-194 \mathrm{~cm}, 21.3-24.8 \mathrm{~kg} / \mathrm{m} 2$ of BMI). The measurements were taken at the Klinisk Fysiologisk Nuklear Medicinsk Afdeling, Herlev-Denmark. Total body potassium (TBK) was measured by ${ }^{40} \mathrm{~K}$ gamma ray detection to determine TBW. Subsequently, after 1 to 3 weeks, total body water (TBW) was measured by bioimpedance (Z) and DXA scan in the same subjects, in the morning after 12 hours rest and with controlled intake in the hospital. The DXA instrument used was a DPX-L bone densitometer 
(LUNAR Corp., Madison, Wisconsin-USA) using photon energies of $38 \mathrm{keV}$ and $70 \mathrm{keV}$. Regions of interest (ROI) were defined manually for each body segment and each scan for the same operator to avoid inter-operator variability. For each segment we obtained fat mass, lean mass and mineral content; from these we obtained TBW assuming a mean hydration for fat and lean body mass (calculated from TBK) of $72 \%$ [8]. The DXA system provided a total trunk volume, and from this we calculated thorax and abdomen volumes assuming an empirical factor used for astronauts [9]. To increase TBW, each subject ingested $3.5 \%$ of their TBW of distilled water, calculated by using the fluid volume estimation obtained previously with ${ }^{40} \mathrm{~K}$. The reference measurement was considered valid if the weight of the volunteer was maintained within $\pm 0.5 \mathrm{~kg}$. Water was administrated at $37^{\circ} \mathrm{C}$ over a period of 30 minutes.

\section{B. Bioimpedance measurement}

We measured 7 segments: head + neck $(\mathrm{HN})$, left-arm (LARM), right-arm (RARM), left-leg (LLEG), right-leg (RLEG), abdomen (AB), thorax (T) and right-side (RS) using 11 disposable electrodes pre-gelled $\mathrm{Ag} / \mathrm{AgCl}$ (ClearTrace 1700-030, Conmed Andover Medical, USA) with the subject in a flat supine position as we shown in Fig. 1. All measurements were made using a 4-electrode method: two electrodes for injecting current (I) and two for sensing volt-

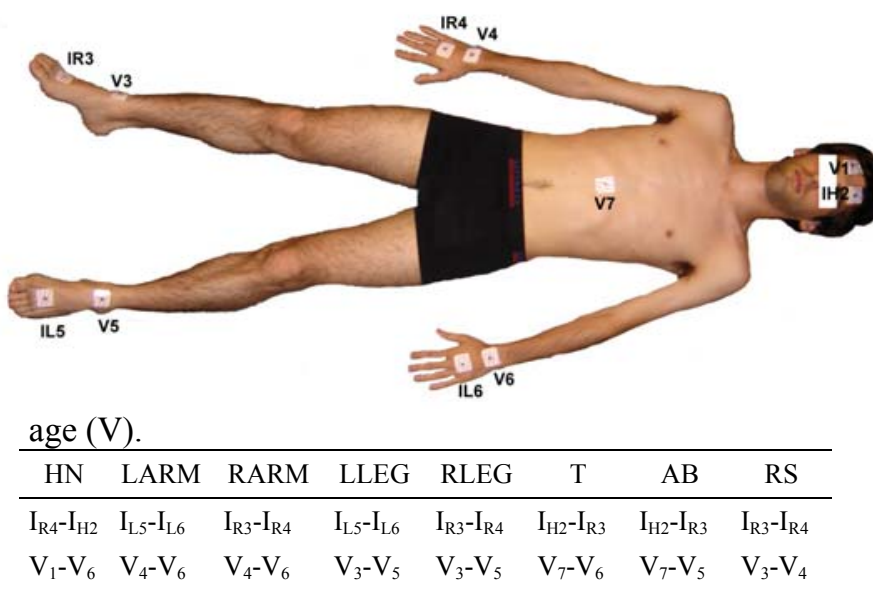

Figure 1. Electrode location for impedance measurement. Injection (I) and voltage detection (V) electrodes are shown for each measured segment

A custom bioimpedance analyzer (MIMsys, NTE, Barcelona, Spain) was employed, with the ability to measure up to 8 selected frequencies in the range of $10 \mathrm{kHz}$ to $1 \mathrm{MHz}$ [10]. We measured at: 10, 20, 50, 100, 500 and $1000 \mathrm{kHz}$. Amplitude of injected current was $300 \mu$ Arms.
Bioimpedance of all segments was measured twice before load and twice after load in order to estimate the short term measurement repeatability.

\section{Total Body Water estimation equations}

To estimate TBW, we used the simplest equation based on the cylindrical model $\mathrm{V}=\mathrm{\rho H}^{2} / \mathrm{R}$ [2]. We excluded the weight in order to allow measurements in microgravity.

We tested other equations, excluding the $\mathrm{H}$ and using the real part of the measured impedance at different frequencies; furthermore, the real part of impedance was extrapolated at infinitum using the Cole dispersion properties [11]. The correlation coefficient between results from different impedance equations and DXA were used for purposes of comparison. The highest correlation $(0.66)$ was found to be for the equation:

$$
T B W=\frac{k_{T B W} \cdot H^{2}}{\operatorname{real}[Z(\infty)]}
$$

where $\mathrm{H}$ is the patient height in $\mathrm{cm}, \mathrm{k}_{\mathrm{TBW}}$ is a gain constant and $\mathrm{Z}(\infty)$ is the impedance at high frequencies estimated using the Cole model with the measurements at 4 different frequencies $(10,20,50$ and $100 \mathrm{kHz})$.

From these results, it is confirmed that the best equation is in the form:

$$
T B W=k o_{T B W}+\frac{k_{T B W} \cdot H^{2}}{\operatorname{real}[Z(\infty)]}
$$

where, $\mathrm{ko}_{\mathrm{TBW}}$ is a constants to adjust for residual volumes not effectively measured.

$$
\text { TWseg }_{i}=k o_{T W \text { segi }}+\frac{k_{T B W} \cdot L_{\text {segi }}^{2}}{\operatorname{real}[Z(\infty)]}
$$

where, $\mathrm{L}_{\text {segi }}$ is an equivalent electrical length for each segment, $\mathrm{k}_{\mathrm{TBW}}$ is gain constants and $\mathrm{ko}_{\mathrm{TW} \text { segi }}$ is residual volumes for each segment. The same approach was used by Bracco et al [6] and Tagliabue et al [12] to calculate segmental fat free mass.

\section{Data Processing}

For each subject, we processed 2 measured in basal state and 2 after fluid load in order to find out mean values and the short term repeatability for each segment. Equations given above were used for all segments and subjects. Individual segments were added to obtain the total water. Standard Errors (SE) were calculated between the expected volume changes, impedance based estimates and volumes given by reference methods.

To compare results using different estimators, the Pearson correlation and the Standard Error SE were used. To 
test the significance of the difference in the variance of errors between different methods de F-Test was used, significance was considered for $\mathrm{P}<0.05$. To describe the dispersion of values for the segmental measurements, the Standard Deviation SD was used. SPSS software version 15.0 (SPSS, Inc.) was used for statistical analysis.

\section{RESUlts}

In table 1, the anthropometric data of each subject before load is given. TBW obtained by DXA and ${ }^{40} \mathrm{~K}$ is compared against TBW assessed by segmental and right-side impedance methods.

Table 1 Subjects' height, weight, TBW estimated using DEXA, ${ }^{40} \mathrm{~K}$, $\mathrm{Z}$ (seg) (adding all measured segments) and Z(RS). Data before load.

\begin{tabular}{lcccccc}
\hline ID & $\begin{array}{c}\mathrm{H} \\
(\mathrm{cm})\end{array}$ & W(kg) & $\begin{array}{c}\text { TBW } \\
{ }^{40} \mathrm{~K}\end{array}$ & $\begin{array}{c}\text { TBW } \\
\text { DEXA }\end{array}$ & $\begin{array}{c}\text { TBW } \\
\text { Z(seg) }\end{array}$ & $\begin{array}{c}\text { TBW } \\
\text { Z(RS) }\end{array}$ \\
\hline 1 & 187 & 77.6 & 41.32 & 41.69 & 41.10 & 41.07 \\
2 & 182 & 74.3 & 38.95 & 38.71 & 39.47 & 39.65 \\
3 & 194 & 80.5 & 38.69 & 40.14 & 41.56 & 41.46 \\
4 & 184 & 84.8 & 41.50 & 41.46 & 39.12 & 38.14 \\
5 & 182 & 80.0 & 41.50 & 42.41 & 43.38 & 44.97 \\
6 & 179 & 74.4 & 41.51 & 41.51 & 43.39 & 44.22 \\
7 & 186 & 79.7 & 45.04 & 40.99 & 41.23 & 41.40 \\
8 & 186 & 84.7 & 44.11 & 43.60 & 43.01 & 44.64 \\
\hline Mean & 185 & 79.5 & 41.58 & 41.31 & 41.53 & 41.94 \\
\hline
\end{tabular}

To estimate the random errors between all the methods for total water assessment, we calculated the Pearson correlation coefficient and the SE between different methods; see table 2 .

Table 2 Correlation coefficients and standard errors for TBW before load between DEXA, ${ }^{40} \mathrm{~K}, \mathrm{Z}(\mathrm{seg})$ and $\mathrm{Z}(\mathrm{RS})$.

\begin{tabular}{lccc}
\hline Pair of estimators & Pearson & SE & SE/mean(\%) \\
\hline Z(seg) vs. Z(RS) & 0.98 & 0.34 & $0.8 \%$ \\
DEXA vs. Z(seg) & 0.63 & 1.22 & $2.9 \%$ \\
DEXA vs. Z(RS) & 0.63 & 1.23 & $2.9 \%$ \\
${ }^{40} \mathrm{~K}$ vs. DEXA & 0.66 & 1.79 & $4.3 \%$ \\
${ }^{40} \mathrm{~K}$ vs. Z(RS) & 0.33 & 2.24 & $5.3 \%$ \\
${ }^{40} \mathrm{~K}$ vs. Z(seg) & 0.31 & 2.25 & $5.4 \%$
\end{tabular}

In table 3 , the expected TBW change (EXP) and the estimated TBW changes due to water load are shown for 4 different estimators: weight of the subject, DXA and impedance using right-side $\mathrm{Z}(\mathrm{RS})$ and the segmental method Z(seg).
Table 3 Comparison of estimated TBW changes produced by the water load using different methods. SE (1) vs EXP for all the other methods.

\begin{tabular}{lccccc}
\hline ID & EXP & W & DEXA & Z(seg) & Z(RS) \\
\hline 1 & 1.45 & 1.7 & 1.69 & 1.47 & 2.48 \\
2 & 1.36 & 1.5 & 1.41 & 0.28 & -1.06 \\
3 & 1.35 & 1.6 & 1.26 & 1.59 & 1.33 \\
4 & 1.45 & 1.7 & 1.01 & 0.73 & 0.63 \\
5 & 1.45 & 1.7 & 1.16 & 1.34 & -0.71 \\
6 & 1.45 & 1.9 & 1.44 & 1.52 & 0.17 \\
7 & 1.57 & 1.4 & 1.85 & 0.62 & 1.69 \\
8 & 1.54 & 1.6 & 1.61 & 0.64 & 0.26 \\
\hline Mean & 1.45 & 1.64 & 1.43 & 1.02 & 0.60 \\
\hline SE (1) & 0.00 & 0.16 & 0.25 & 0.53 & 1.24 \\
\hline
\end{tabular}

Compared to expected values, the best estimator (lower SE) for TBW was weight, followed by DXA and the two impedance methods: $Z($ seg) and $Z(R S)$. The biggest dispersion of TBW increment, compared with all other methods, was when the Right-Side impedance $\mathrm{Z}(\mathrm{RS})$ was used $\mathrm{SE}=1.241$.

Table 4 Mean and standard deviation of water increment after load (1) for each segment: expected value EXP, DXA and Z methods. Last two columns are the maximum differences between each method and the expected result in each segment.

\begin{tabular}{|l|c|r|r|r|r|r|r|}
\cline { 2 - 8 } \multicolumn{1}{c|}{} & \multicolumn{3}{c|}{$\begin{array}{c}\text { Water } \\
\text { Increment (1) }\end{array}$} & \multicolumn{2}{c|}{$\begin{array}{c}\text { Standard } \\
\text { Deviation (1) }\end{array}$} & \multicolumn{2}{c|}{$\begin{array}{c}\text { Maximum } \\
\text { difference (1) }\end{array}$} \\
\cline { 2 - 8 } \multicolumn{1}{c|}{} & EXP & DXA & Z & $\begin{array}{c}\text { DXA- } \\
\text { EXP }\end{array}$ & $\begin{array}{c}\text { Z- } \\
\text { EXP }\end{array}$ & $\begin{array}{c}\text { DXA- } \\
\text { EXP }\end{array}$ & $\begin{array}{c}\text { Z- } \\
\text { EXP }\end{array}$ \\
\hline Head & 0,10 & 0,04 & 0,05 & 0,12 & 0,07 & 0,23 & 0,19 \\
L. Arm & 0,09 & $-0,07$ & 0,08 & 0,24 & 0,02 & 0,56 & 0,08 \\
R. Arm & 0,09 & 0,00 & 0,02 & 0,43 & 0,04 & 0,71 & 0,13 \\
L. Leg & 0,26 & $-0,10$ & 0,08 & 0,67 & 0,04 & 1,76 & 0,26 \\
R. Leg & 0,27 & $-0,02$ & 0,09 & 0,65 & 0,10 & 1,77 & 0,29 \\
Thorax & 0,29 & 0,62 & 0,42 & 0,56 & 0,24 & 1,58 & 0,47 \\
Abdomen & 0,36 & 0,77 & 0,27 & 0,69 & 0,14 & 1,97 & 0,47 \\
\hline
\end{tabular}

Total water for all segments and subjects were calculated using DXA and segmental bio-impedance measurements. If a uniform distribution of water is assumed in all the segments, an increment of $3.5 \%$ must appear in all of them. Table 4 shows expected water increments and mean values estimated with DXA and Z. Maximum individual errors between the expected increment and DXA was 1.971 for the abdomen while for $\mathrm{Z}$ was only $0.47 \mathrm{l}$. In all the segments the impedance method had lower differences compared to expected values; see last two columns of table 4 . 


\section{Discussion}

The reference methods used for validation (DXA and ${ }^{40} \mathrm{~K}$ ) were expected to have a better than $6 \%$ accuracy [13] for the estimation of total lean body mass or water. The correlation for TBW between both methods was 0.66 , with an SE of 1.791 (4.3\% of TBW, table 2$)$, showing the existence of random errors between the two methods in the expected accuracy range. The SE increased up to 2.251 between ${ }^{40} \mathrm{~K}$ and impedance, and the lower error was obtained between Z(RS) and Z(seg) with a SE of 0.341 (table 2 ). The best agreement between DXA and impedance is explained by the fact that impedance equations were adjusted using mean DXA results. These results indicate that random errors in the estimation of TBW using ${ }^{40} \mathrm{~K}$, DXA and impedance methods are in the same order of magnitude.

The results between DXA and right-side impedance are in agreement with previous results in a healthy and nonobese population Erselcant et al [14].

The analysis of the results in the determination of water changes after load (table 3 ) shows that all the methods were close to the expected water increment of 1.451 , except for $\mathrm{Z}(\mathrm{RS})$, which underestimated the water increment by 0.51 ($34 \%$ ). The advantage of employing $Z(\mathrm{seg})$ over $Z(R S)$ is based on the comparison of the SE for both methods using the expected water increment as a reference. In the water load experiment, the SE using Z(seg) is significantly lower than using $\mathrm{Z}(\mathrm{RS})$ in the estimation of TBW changes (F-test, $\mathrm{p}<0.05)$.

When estimating segmental water changes, DXA showed higher discrepancies than $\mathrm{Z}$ compared to expected increments (table 4). This results are based on a uniform distribution of water in all the body; the protocol was designed to give enough time to rich a uniform distribution of water but not direct prove of this was obtained. Taking into account individual values, DXA and $Z$ showed high variance with errors up to 21 for DXA and 0.51 for $Z$ when measuring the abdomen.

\section{ConClusion}

The segmental method had lower standard error $(0.51)$ than the right side method (1.24 1) in the estimation of small increments of TBW compared to expected values.

The segmental impedance method is able to determine segmental increments of water with lower mean error and standard deviation than DXA method. Changes in water segments estimated by impedance where more close to expected values than using DXA.

The results are representative of the capabilities and the limits of impedance methods when used in a healthy popu- lation with a comparable anthropometry. These methods could be used in space or other situations, for example, in sport medicine or aeronautics due to its simplicity and the low volume and weight of the required measurement instrumentation.

\section{ACKNOWLEDGMENT}

This work was supported by Nuevas Tecnologias del Espacio (NTE) S.A. and the European Space Agency, contract: $11299 / 94 / \mathrm{NL} / \mathrm{FB}$.

\section{REFERENCES}

1. Schwan HP (1957) Electrical properties of tissue and cell suspensions. In: Lawrence JH. Tobias CA (eds) Advances in biological and medical physics. New York, Academic Press, vol.V, 147-209

2. Hoffer EC, Meador CK, and Simpson DC (1969) Correlation of whole-body impedance with total body water volume J. Appl. Physiol. 27: 531-534

3. Ellis KJ 2000 Human body composition: in vivo methods Phyiol. Rev. 80: 649-680

4. Nyboer J (1972) Workable volume and flow concepts of biosegments by electrical impedance plethysmography Tower Int. Technomed. J. Life Sci. 2: 1-13

5. Patterson R, Ranganathan C, Engel R and Berkseth R (1988) Measurement of body fluid volume change using multi-site impedance measurements Med. Biol. Eng. Comput. 26: 33-37

6. Bracco D, Thiébaud D, Chioléro RL (1996) Segmental body composition assessed by bioelectrical impedance analysis and DEXA in humans J Appl. Physiol. 81(6): 2580-2587

7. Rosell Ferrer J, Riu Costa P, Pallas Areny R. et al (2000) Apparatus and procedure for measuring volumes and global and segmental corporal composition in human beings. US Patent office. Patent number. US6151523

8. Wang Z, Deurenberg P, Wang W, Pietrobelli A, et al (1999) Hydration of fat-free mass: new physiological modeling approach Am. J. Physiol. Endocrinol. Metab. 276: E995-E1003

9. International Space Station, Flight crew integration standard, SSP50005, rev B, 1995

10. Lozano A, Rosell J and Pallas-Areny R (1995) A multifrequency multichannel electrical impedance data acquisition system for body fluid shift monitoring Physiol. Meas. 16: 227-237

11. Cole KS. Electric impedance of suspensions of spheres J (1928) Gen. Physiol. 1928, 12: 29-36

12. Tagliabue A, Andreoli A, Comelli M, Bertoli S, et al (2001) Prediction of lean body mass from multifrequency segmental impedance: influence of adiposity Acta Diabetol. 38: 93-97

13. Mattsson S, Thomas BJ (2006) Development of methods for body composition studies Phys Med Biol 51: R203-R228

14. Erselcant T, Candan F, Saruhan S, Ayca T (2000) Comparison of Body Composition Analysis Methods in Clinical Routine Annals of Nutrition \& Metabolism 44: 243-248

Author: Javier Rosell-Ferrer

Institute: Technical University of Catalonia

Street: Jordi Girona, 1-3, Edifici C4

City: 08034 Barcelona

Country: Spain

Email: jrosell@eel.upc.edu 